\title{
BLENDING METHODS FOR COUPLING ATOMISTIC AND CONTINUUM MODELS
}

\author{
P. Bochev, R. Lehoucq, M. Parks, S. Badia, and M. Gunzburger
}

\subsection{Introduction}

In this paper we review recent developments in blending methods for atomisticto-continuum (AtC) coupling in material statics problems. Such methods tie together atomistic and continuum models by using a bridge domain that connects the two models. There are several reasons why AtC coupling methods (of which blending methods are a subset) are important and have been subject to an increased interest in the recent years. Despite tremendous increases in computational power, fully atomistic simulations on an entire model domain remain computationally infeasible for many applications of interest. As a result, attention has focused on hybrid schemes where in all regions with well-behaved solutions, the atomistic (microscopic) model is replaced by a (macroscopic) continuum model enabling a more efficient computational scheme (see [14, 29, 9] for general information). The main challenge is the synthesis of the two distinct models in a manner that minimizes, or altogether eliminates, undesirable artifacts such as ghost forces, unphysical solutions let alone supporting mathematical analysis. Notable AtC coupling methods include the quasicontinuum method [39], the bridging scale decomposition [41], and [24] where atomistic and continuum models are overlapped (see the latter two references, and those mentioned above for numerous citations to the literature). The numerical analysis of AtC methods has lagged in comparison to the number of methods proposed; see the recent papers $[2,3,17,31,26,27]$ for analyses of the quasicontinuum method.

Blending methods couple atomistic/continuum modes via a dedicated blending, or bridge, region inserted between the atomistic and continuum subdomains. The atomistic and continuum models are tied together by using a suitable "continuity" condition (or balance law) for the atomistic and continuum positions or displacements in this region. A complicating factor is that the atomistic and (classical) continuum elastic models rely on nonlocal and local models of force interaction, respectively. In the (classical) elastic context, the "local force" is that exerted on a body by contact forces occurring on the surface (of the body). In contrast, in the atomistic model, forces are summed from atoms separated by a finite distance. The incompatibility arising from coupling local and nonlocal force models is intrinsic. The goal of our paper is threefold. First, we review how blending approaches attempt to ameliorate the various negative effects by relying on an interface between the two models. Second, we discuss the beginnings of a 
numerical analysis by discussing consistency of the resulting numerical schemes. Third, we suggest how the intrinsic limitation associated with coupling nonlocal and local mechanical theories may be avoided by replacing the classical elastic theory with a nonlocal elastic theory.

The use of a bridge domain in blending methods bears a resemblance to conventional overlapping domain decomposition schemes (see [33, 40] for discussions and references to the literature). However, blending methods are more complicated because they combine two mathematically distinct descriptions of the material response, and their main goal is to reconcile these descriptions over a transitional region. To fix the main ideas below we briefly summarize the basic concepts of AtC blending methods.

\subsubsection{An overview of AtC blending methods}

Suppose that we want to find the deformed configuration at zero temperature of a material that occupies a bounded region $\Omega$ in a two- or three-dimensional Euclidean space. Assume that we have two different mathematical models of this material: an atomistic and continuum described by atomistic and continuum operators $\mathcal{L}^{a} \mathcal{L}^{c}$, respectively. Finally, assume that the problem configuration is such that

- the atomistic model is valid throughout $\Omega$

- solving the atomistic problem on all of $\Omega$ is prohibitively expensive

- the continuum model is valid in a subregion $\Omega^{\prime} \subset \Omega$

- the continuum operator $\mathcal{L}^{c}$ approximates well (in a suitable sense) the atomistic operator in $\Omega^{\prime}$, but is not valid ${ }^{1}$ in $\Omega_{a}=\Omega \backslash \Omega^{\prime}$.

The last two assumptions define the scope of hybrid methods: they are unnecessary if the continuum model remains valid throughout $\Omega$, and they are not appropriate unless atomistic and continuum models are "close" on some part of the domain.

In particular, an AtC blending method partitions $\Omega^{\prime}$ into nonoverlapping continuum region $\Omega_{c}$ and a blending region $\Omega_{b}$ so that $\Omega=\Omega_{a} \cup \Omega_{b} \cup \Omega_{c}$; see Figure 6.1. The basic idea is to avoid the computational expense associated with solving the atomistic equations on all of $\Omega$ by using instead

- the continuum model in $\Omega_{c}$ (where it is assumed valid)

- the atomistic model in $\Omega_{a}$ (where the continuum model is assumed invalid)

- a blending of the two models over the bridge region $\Omega_{b}$.

The cost of an ensuing computational method is minimized when $\Omega_{a}$ and $\Omega_{b}$ are small relative to $\Omega_{c}$.

A typical AtC blending method has four main ingredients:

- blending functions $\theta_{a}$ and $\theta_{c}$ that form a partition of unity on $\Omega$

- an operator $\mathcal{L}_{\theta}^{a}$ acting on $\Omega_{a} \cup \Omega_{b}$, and such that $\left.\mathcal{L}_{\theta}^{a}\right|_{\Omega_{a}}=\mathcal{L}^{a}$

${ }^{1}$ By this we mean that the physical phenomena in $\Omega^{\prime}$ cannot be modeled well by $\mathcal{L}^{c}$. 

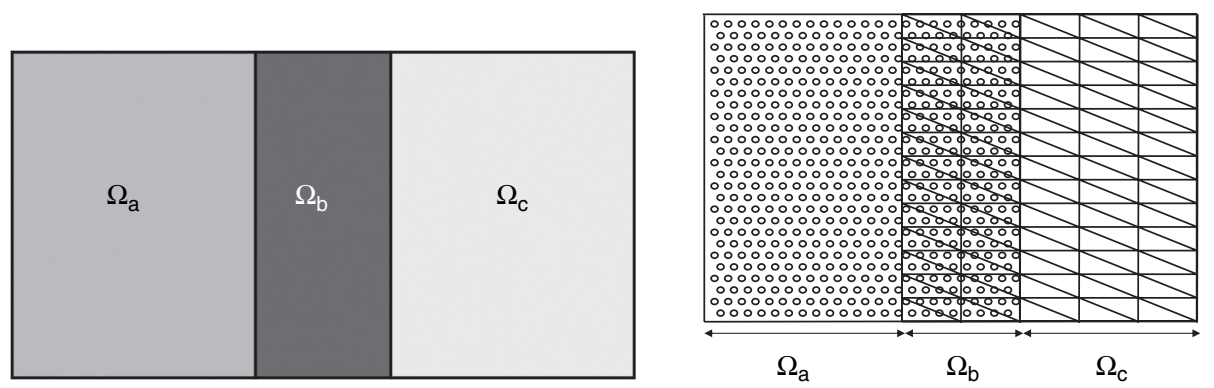

Figure 6.1: Typical domain configurations for AtC blending methods. The left plot depicts the original problem domain $\Omega$ and its partition into an atomistic domain $\Omega_{a}$, continuum domain $\Omega_{c}$, and a bridge domain $\Omega_{b}$. The right plots depict the atomic lattice in $\Omega_{a} \cup \Omega_{b}$ and a finite element mesh in $\Omega_{b} \cup \Omega_{c}$ for the continuum model.

- an operator $\mathcal{L}_{\theta}^{c}$ acting on $\Omega_{b} \cup \Omega_{c}$, and such that $\left.\mathcal{L}_{\theta}^{c}\right|_{\Omega_{c}}=\mathcal{L}^{c}$

- an operator $\mathcal{C}$ acting on $\Omega_{b}$.

Operators $\mathcal{L}_{\theta}^{a}$ and $\mathcal{L}_{\theta}^{c}$ are blended versions of the original atomistic and continuum operators $\mathcal{L}^{a}$ and $\mathcal{L}^{c}$, defined using the blending functions. Their purpose is to avoid "duplication" of the material response in the bridge region that results from simply superimposing the two models in $\Omega_{b} . \mathcal{C}$ is a constraint operator that enforces the "continuity" between atomistic and continuum solutions in the bridge region.

AtC blending methods are a relatively recent development that is driven by simulation needs in nanotechnology and material sciences. These simulation needs have resulted in the creation of many ad hoc AtC blending methods that are often loosely defined and focused on specific problems. This makes the numerical analysis of blending methods difficult. Depending on the atomistic and continuum models used in the AtC method $\mathcal{L}^{a}$ and $\mathcal{L}^{c}$ may correspond to a force equilibrium or an energy minimization principle. In the first case these operators are related to Newton's second law and we talk about force-based AtC blending. Examples of force-based blending methods derived from mechanical arguments are given in $[4,5,20]$. In the second case AtC blending is energy-based. A representative example of an energy-based method, defined by blending of atomistic and continuum energy functionals, can be found in [8]. One-dimensional results and analysis for blending harmonic potentials and linear elasticity via the Arlequin method [16] is the subject of $[6,34]$. The overlapping AtC method presented in [24] can also be considered a blending method, and demonstrates how ghost forces can be eliminated by considering a patch test. We also remark that blending methods have been introduced within the context of meshfree methods [22] where the atomistic region is replaced by a region discretized with a meshfree method. 
Our paper reviews an abstract framework for force- or energy-based AtC blending methods that includes precise notions of patch and consistency tests. Our discussion on energy-based blending methods is new work. The framework allows us to identify four general classes of AtC methods, explains the origin of so-called ghost forces, and the satisfaction of Newton's third law.

We have organized the paper as follows. The model atomistic and continuum problems are introduced in Section 6.2. Section 6.3 discusses force-based AtC blending methods and their taxonomy, and states formal definitions of consistency and patch test. Energy-based AtC blending methods and their taxonomy are briefly discussed in Section 6.4. Our conclusions are summarized in Section 6.6.

\subsubsection{Notation}

The following notation is used throughout the paper. Double fonts $(\mathbb{A})$ denote sets of atoms, except for $\mathbb{R}^{d}$ and $\mathbb{R}$ that stand for $d$-dimensional and 1-dimensional Euclidean spaces, respectively. Standard upper-case fonts $(A)$ are used for atomistic and continuum spaces, calligraphic fonts $(\mathcal{L})$ for operators and functionals, lower-case bold letters $(\boldsymbol{a})$ for vector-valued continuum functions, lower-case bold Greek letters $(\boldsymbol{\alpha})$ for vector-valued atomistic (discrete) functions and Lagrange multipliers.

A superscript in the space designation indicates the support of the functions in this space and a subscript shows the type of boundary constraint imposed on its elements. For example, the elements of $X^{a b}$ are supported on $\Omega_{a} \cup \Omega_{b}$, the elements of $X_{0}$ vanish on the boundary, and $X_{D}$ is an affine space whose elements are subject to an inhomogeneous boundary condition. A lack of superscript, e.g., $X$, indicates that the elements of the space are supported on all of $\Omega$. Likewise, absence of a subscript means that the space is not constrained by boundary conditions.

Dual spaces are denoted by $(\cdot)^{\prime}$. In general, discrete and continuous $L^{2}$ inner products, $L^{2}$ norms, and duality pairings are denoted by $(\cdot, \cdot),\|\cdot\|$, and $\langle\cdot, \cdot\rangle$, respectively. Additional notation will be introduced as needed.

\subsection{Atomistic and continuum models}

This section provides a brief summary of the basic atomistic and continuum material statics models that will be used in the paper. Because our main focus is on mathematical aspects of AtC blending, rater than material modeling, we intentionally choose the simplest possible material models.

\subsubsection{Force-based models}

Force-based atomistic and continuum models are derived by equilibrating internal and applied forces at each atom or material point. Material properties in this case are encoded in terms of internal force operators. For continuum materials internal forces are usually described by partial differential operators, although other (non-local, integral) choices are also possible, as discussed in Section 6.5. 


\subsubsection{The atomistic model}

Let $\mathbb{P}$ denote an undeformed (or reference) lattice of $|\mathbb{P}|$ identical particles located in a bounded, Euclidean region $\Omega \subset \mathbb{R}^{d}$. The spatial position vectors of the particle $\alpha \in \mathbb{P}$ in the undeformed and deformed configurations are $\boldsymbol{x}_{\alpha}$ and $\boldsymbol{q}_{\alpha}$, and $\boldsymbol{\psi}_{\alpha}=\boldsymbol{q}_{\alpha}-\boldsymbol{x}_{\alpha}$ is the displacement vector of $\alpha$. The set of all possible atomistic displacements is a finite dimensional space $X$ whose elements $\phi \in X$ are sets of properly ordered $|\mathbb{P}| \times d$ scalar values $\phi_{\alpha}^{i}$ for $\alpha \in \mathbb{P}$ and $i=1, \ldots, d$.

Let $\mathbb{D} \subset \mathbb{P}$ denote the subset of particles whose positions in the deformed configuration are fixed. We define the affine space

$$
X_{D}:=\left\{\phi \in X \mid \phi_{\alpha}=\psi_{\alpha}^{\mathbb{D}} \forall \alpha \in \mathbb{D}\right\}
$$

where $\boldsymbol{\psi}_{\alpha}^{\mathbb{D}}$ is the given, fixed displacement vector, and the subspace

$$
X_{0}:=\left\{\phi \in X \mid \phi_{\alpha}=\mathbf{0} \forall \alpha \in \mathbb{D}\right\}
$$

of $X$ in which all particles from $\mathbb{D}$ have zero displacements. These spaces are atomistic counterparts of Sobolev spaces constrained by inhomogeneous and homogeneous Dirichlet boundary conditions for a continuum problem.

The strong form of the lattice statics problem consists of finding an equilibrium (deformed) configuration $\left\{\boldsymbol{\psi}_{\alpha}\right\}_{\alpha \in \mathbb{P} \backslash \mathbb{D}}$ which satisfies the force-balance problem

$$
\begin{aligned}
\left(\mathcal{L}^{a}(\boldsymbol{\psi})\right)_{\alpha}+\chi_{\alpha} & =\mathbf{0} & & \forall \alpha \in \mathbb{P} \backslash \mathbb{D} \\
\boldsymbol{\psi}_{\alpha} & =\boldsymbol{\psi}_{\alpha}^{\mathbb{D}} & & \forall \alpha \in \mathbb{D}
\end{aligned}
$$

In $(6.1 \mathrm{a})\left(\mathcal{L}^{a}(\cdot)\right)_{\alpha}$ and $\chi_{\alpha}$ are the internal and external forces acting on the particle $\alpha$, respectively. Therefore, the atomistic problem (6.1) is simply Newton's second law for a system of particles interacting via the force operator $\mathcal{L}^{a}$ and the applied forces $\chi$, and constrained to satisfy the "boundary condition" (6.1b). Note that $\mathcal{L}^{a}: X \rightarrow X$ is generally a non-linear operator.

A weak formulation of (6.1) is as follows: find $\psi \in X_{D}$ such that

$$
\mathcal{B}^{a}(\boldsymbol{\psi}, \phi)=\mathcal{G}^{a}(\phi) \quad \forall \phi \in X_{0},
$$

where

$$
\mathcal{B}^{a}(\boldsymbol{\psi}, \boldsymbol{\phi})=\left(\mathcal{L}^{a}(\boldsymbol{\psi}), \boldsymbol{\phi}\right) \quad \text { and } \quad \mathcal{G}^{a}(\phi)=-(\boldsymbol{\chi}, \boldsymbol{\phi}) \quad \text { for } \boldsymbol{\psi} \in X, \boldsymbol{\phi} \in X_{0}
$$

Equation (6.2) is the principle of virtual work. While it is similar in form to variational equations arising from continuum models, it is important to keep in mind that force balance equations (6.1a) are inherently non-local in nature, i.e., in general, the force acting on a particle $\alpha \in \mathbb{P} \backslash \mathbb{D}$ depends on the displacements of many other particles separated by a finite distance. 


\subsubsection{The continuum model}

Let $\boldsymbol{x}, \boldsymbol{q}$, and $\boldsymbol{u}=\boldsymbol{q}-\boldsymbol{x}$ be the spatial position vectors in the undeformed and deformed continuum configurations, and the continuum displacement vector, respectively. The strong form of the continuum model is

$$
\begin{aligned}
\mathcal{L}^{c}(\boldsymbol{u}) & =\boldsymbol{f} & & \text { in } \Omega, \\
\boldsymbol{u} & =\boldsymbol{u}^{\partial \Omega} & & \text { on } \partial \Omega,
\end{aligned}
$$

where $\mathcal{L}^{c}$ denotes a (possibly nonlinear) differential operator, $\boldsymbol{f}$ the external force, $\partial \Omega$ the boundary of $\Omega$, and $\boldsymbol{u}^{\partial \Omega}$ the prescribed boundary data. ${ }^{2}$ In what follows we assume that (6.4) is a local model in the sense that the stress at a point $\boldsymbol{x} \in \Omega$ depends only on the values of $\boldsymbol{u}$ and $\nabla \boldsymbol{u}$ at that point. We also assume that there exist differential operators $\mathcal{L}_{S}^{c}(\cdot)$ and $\mathcal{L}_{E}^{c}(\cdot)$ such that

$$
\left\langle\mathcal{L}^{c}(\boldsymbol{u}), \boldsymbol{v}\right\rangle=\int_{\Omega} \mathcal{L}_{S}^{c}(\boldsymbol{u}): \mathcal{L}_{E}^{c}(\boldsymbol{v}) d \boldsymbol{x}
$$

for all smooth functions $\boldsymbol{u}$ and $\boldsymbol{v}$ with $\boldsymbol{v}=\mathbf{0}$ on $\partial \Omega ;(\cdot):(\cdot)$ denotes the scalar tensor product operation. To state the weak form of (6.4) let $Y$ denote a Hilbert space, defined with respect to $\Omega$, and such that for any $\boldsymbol{v} \in Y, \mathcal{L}_{S}^{c}(\boldsymbol{v})$ and $\mathcal{L}_{E}^{c}(\boldsymbol{v})$ are meaningful in $L^{2}$ sense. We define the affine subspace and subspace

$$
Y_{D}:=\left\{\boldsymbol{v} \in Y \mid \boldsymbol{v}=\boldsymbol{u}^{\partial \Omega} \text { on } \partial \Omega\right\} \quad \text { and } \quad Y_{0}:=\{\boldsymbol{v} \in Y \mid \boldsymbol{v}=\mathbf{0} \text { on } \partial \Omega\},
$$

respectively, and the functionals

$$
\mathcal{B}^{c}(\boldsymbol{u}, \boldsymbol{v}):=\int_{\Omega} \mathcal{L}_{S}^{c}(\boldsymbol{u}): \mathcal{L}_{E}^{c}(\boldsymbol{v}) d \boldsymbol{x} \quad \text { and } \quad \mathcal{G}^{c}(\boldsymbol{v})=\langle\boldsymbol{f}, \boldsymbol{v}\rangle \quad \text { for } \boldsymbol{u} \in Y, \boldsymbol{v} \in Y_{0} .
$$

Then, a weak formulation of (6.4) is: given $\boldsymbol{f} \in\left(Y_{0}\right)^{\prime}$, find $\boldsymbol{u} \in Y_{D}$ such that

$$
\mathcal{B}^{c}(\boldsymbol{u}, \boldsymbol{v})=\mathcal{G}^{c}(\boldsymbol{v}) \quad \forall \boldsymbol{v} \in Y_{0} .
$$

Note that, as a rule, the operator $\mathcal{L}_{E}^{c}(\cdot)$ is linear but, in general, $\mathcal{L}_{S}^{c}(\cdot)$ is nonlinear.

\subsubsection{Energy-based models}

Energy-based models in material statics postulate that the equilibrium (deformed) configuration of an atomistic or a continuum system minimizes the potential energy of the system. For these models material properties are encoded in terms of potential energy functionals.

\footnotetext{
${ }^{2}$ For simplicity, we consider only Dirichlet boundary conditions.
} 


\subsubsection{The atomistic model}

We retain the notation from the force-based atomistic model. In particular, $\mathbb{P}$ is an undeformed lattice of identical particles in $\Omega$ and $\boldsymbol{\psi}_{\alpha}$ is the displacement of the particle $\alpha$. The (atomistic) potential energy of the particle system is given by the expression

$$
\mathcal{E}^{a}(\boldsymbol{\psi})=\sum_{\alpha \in \mathbb{P}}\left(W_{\alpha}^{a}(\boldsymbol{\psi})+\chi_{\alpha} \cdot \boldsymbol{\psi}_{\alpha}\right)
$$

where $W_{\alpha}^{a}(\cdot)$ and $\chi_{\alpha}$ denote the potential energy associated with the particle $\alpha$, and the external force applied to that particle. Note that $W_{\alpha}^{a}$ may depend on the displacements of all the particles $\boldsymbol{\psi}_{\beta}, \beta \in \mathbb{P}$, although in many cases it will depend only on the displacement of the particles within some ball $\mathcal{B}_{\alpha}=\{\mathbf{x} \in$ $\left.\Omega:\left|\mathbf{x}-\mathbf{x}_{\alpha}\right| \leq r\right\}$ for some given $r>0$. As an example, we could set $W_{\alpha}^{a}$ to the Leonard-Jones potential.

The equilibrium deformed atomistic configuration $\boldsymbol{\psi}$ is characterized by

$$
\psi=\arg \min _{\phi \in X_{D}} \mathcal{E}^{a}(\phi)
$$

As a result, $\boldsymbol{u}$ is subject to the first-order necessary optimality condition

$$
\delta_{\boldsymbol{\psi}} \mathcal{E}^{a}(\boldsymbol{\psi}) \equiv \lim _{\epsilon \rightarrow 0} \frac{d}{d \epsilon} \mathcal{E}^{a}(\boldsymbol{\psi}+\epsilon \boldsymbol{\phi})=0 \quad \forall \boldsymbol{\phi} \in X_{0}
$$

This problem can be further replaced by a weak variational formulation that has the same form as (6.2).

\subsubsection{The continuum model}

Suppose that $\Omega$ is occupied by a continuum material with a prescribed boundary displacement $\boldsymbol{u}^{\partial \Omega}$, and whose potential energy at a point $\boldsymbol{x}$ in the deformed configuration is given by $W^{c}(\boldsymbol{u})$. Then, the total potential energy associated with this material is

$$
\mathcal{E}^{c}(\boldsymbol{u})=\int_{\Omega}\left(W^{c}(\boldsymbol{u})+\mathbf{f} \cdot \boldsymbol{u}\right) d \Omega
$$

where $\mathbf{f}$ is the external volumetric force applied at each point in $\Omega$. The equilibrium deformed configuration is characterized by an energy principle similar to $(6.8)$,

$$
\boldsymbol{u}=\arg \min _{\boldsymbol{v} \in X_{D}} \mathcal{E}^{c}(\boldsymbol{v})
$$

and is subject to an analogous first-order optimality condition:

$$
\delta \boldsymbol{u} \mathcal{E}^{c}(\boldsymbol{u}) \equiv \lim _{\epsilon \rightarrow 0} \frac{d}{d \epsilon} \mathcal{E}^{c}(\boldsymbol{u}+\epsilon \boldsymbol{v})=0 \quad \forall \boldsymbol{v} \in Y_{0} .
$$


This problem also can be replaced by a weak variational equation that assumes the same form as (6.6). As an example, we could set $W^{c}(\boldsymbol{u})=\sigma(\boldsymbol{u}): \varepsilon(\boldsymbol{u})$ where $\sigma$ and $\varepsilon$ denote the work conjugate stress and strain tensors, respectively. Then, minimizers of (6.11) solve the weak equation: seek $\boldsymbol{u} \in Y_{D}$ such that

$$
\int_{\Omega} \sigma(\boldsymbol{u}): \varepsilon(\boldsymbol{v}) d \Omega=\int_{\Omega} \mathbf{f} \cdot \boldsymbol{v} d \Omega \quad \forall \boldsymbol{v} \in Y_{0} .
$$

With $\mathcal{L}_{S}^{c}(\cdot)=\sigma(\cdot)$ and $\mathcal{L}_{E}^{c}(\cdot)=\varepsilon(\cdot)$, the abstract weak problem (6.6) reduces to $(6.13)$.

\subsection{Force-based blending}

This section reviews an AtC blending method to merge force-based atomistic and continuum material models. The blending process is effected by using the weak forms (6.2) and (6.6) of the models. General definitions of consistency and patch tests for AtC methods are also reviewed.

\subsubsection{An abstract AtC blending method}

Let $\mathbb{A}, \mathbb{B}$, and $\mathbb{C}$ denote the particles associated with $\Omega_{a}, \Omega_{b}$, and $\Omega_{c}$, respectively; particles on the interfaces between $\Omega_{b}$ and the other two subdomains are assigned to $\mathbb{B}$. We introduce the atomistic spaces

$$
X_{0}^{a b}:=\left\{\left.\left(\phi_{\alpha}\right)\right|_{\alpha \in \mathbb{A} \cup \mathbb{B}} \mid \phi \in X_{0}\right\} \quad \text { and } \quad X_{D}^{b}:=\left\{\left.\left(\phi_{\alpha}\right)\right|_{\alpha \in \mathbb{B}} \mid \phi_{\alpha} \in X_{D}\right\},
$$

the continuum subspace

$$
Y_{0}^{b c}:=\left\{\left.\boldsymbol{v}\right|_{\Omega_{b} \cup \Omega_{c}} \mid \boldsymbol{v} \in Y_{0}\right\}
$$

and the continuum affine subspaces

$$
Y_{D}^{b c}:=\left\{\left.\boldsymbol{v}\right|_{\Omega_{b} \cup \Omega_{c}} \mid \boldsymbol{v} \in Y_{D}\right\} \quad \text { and } \quad Y_{D}^{b}:=\left\{\left.\boldsymbol{v}\right|_{\Omega_{b}} \mid \boldsymbol{v} \in Y_{D}\right\} .
$$

A force-based AtC blending method has the following key ingredients:

1. atomistic and continuum blending functions $\theta_{a}$ and $\theta_{c}$, respectively, such that $\theta_{a} \geq 0, \theta_{c} \geq 0, \theta_{a}=1$ in $\Omega_{a}, \theta_{c}=1$ in $\Omega_{c}$ and $\theta_{a}+\theta_{c}=1$ in $\Omega$;

2. a constraint operator $\mathcal{C}(\cdot, \cdot): X_{D}^{b} \times Y_{D}^{b} \rightarrow Q$, where $Q$ is a function space whose definition depends on the particular nature of the constraints.

3. blended atomistic functionals $\mathcal{B}_{\theta}^{a}\left(\cdot, \cdot ; \theta_{a}\right): X_{D} \times X_{0}^{a b} \rightarrow \mathbb{R}$ and $\mathcal{G}_{\theta}^{a}\left(\cdot ; \theta_{a}\right)$ : $X_{0}^{a b} \rightarrow \mathbb{R}$ such that

$\mathcal{B}_{\theta}^{a}(\boldsymbol{\psi}, \boldsymbol{\phi} ; 1)=\mathcal{B}^{a}(\boldsymbol{\psi}, \boldsymbol{\phi})$ and $\mathcal{G}_{\theta}^{a}(\boldsymbol{\phi} ; 1)=\mathcal{G}^{a}(\boldsymbol{\phi})$ for all $\{\boldsymbol{\psi}, \boldsymbol{\phi}\} \in X_{D} \times X_{0}^{a b} ;$

4. blended continuous functionals $\mathcal{B}_{\theta}^{c}\left(\cdot, \cdot ; \theta_{c}\right): Y_{D}^{b c} \times Y_{0}^{b c} \rightarrow \mathbb{R}$ and $\mathcal{G}_{\theta}^{c}\left(\cdot ; \theta_{c}\right)$ : $Y_{0}^{b c} \rightarrow \mathbb{R}$ such that

$\mathcal{B}_{\theta}^{c}(\boldsymbol{u}, \boldsymbol{v} ; 1)=\mathcal{B}^{c}(\boldsymbol{u}, \boldsymbol{v})$ and $\mathcal{G}_{\theta}^{c}(\boldsymbol{v} ; 1)=\mathcal{G}^{c}(\boldsymbol{v})$ for all $\{\boldsymbol{u}, \boldsymbol{v}\} \in Y_{D}^{b c} \times Y_{0}^{b c} ;$ 
Using these definitions, an abstract, force-based AtC blending method can be expressed in the following form: find $\{\boldsymbol{\psi}, \boldsymbol{u}\} \in X_{D} \times Y_{D}^{b c}$ such that

$$
\left\{\begin{aligned}
\mathcal{B}_{\theta}^{a}\left(\boldsymbol{\psi}, \boldsymbol{\phi} ; \theta_{a}\right)+\mathcal{B}_{\theta}^{c}\left(\boldsymbol{u}, \boldsymbol{v} ; \theta_{c}\right)= & \mathcal{G}_{\theta}^{a}\left(\boldsymbol{\phi} ; \theta_{a}\right)+\mathcal{G}_{\theta}^{c}\left(\boldsymbol{v} ; \theta_{c}\right) \forall\{\boldsymbol{\phi}, \boldsymbol{v}\} \in X_{0}^{a b} \times Y_{0}^{b c} \\
& \text { subject to } \\
\boldsymbol{\psi}_{\alpha}=\boldsymbol{u}\left(\boldsymbol{x}_{\alpha}\right) \forall \alpha \in \mathbb{C} \backslash(\mathbb{C} \cap \mathbb{D}) & \text { and } \mathcal{C}(\boldsymbol{\psi}, \boldsymbol{u})=0 \forall\{\boldsymbol{\psi}, \boldsymbol{u}\} \in X_{D}^{b} \times Y_{D}^{b}
\end{aligned}\right.
$$

The first equation in (6.14) is a weak blended force-balance equation. The second equation is a constraint which states that atomistic displacements in $\Omega_{c}$ are slaved to the continuum displacements, and the last equation is a constraint that ties the two models together over the blend region. A particular AtC blending method results from choosing the four key ingredients above, i.e., the blending functions and definitions of the functionals $\mathcal{B}_{\theta}^{a}, \mathcal{B}_{\theta}^{c}, \mathcal{G}_{\theta}^{c}, \mathcal{G}_{\theta}^{a}$, and $\mathcal{C}$.

Note that in (6.14) the atomistic test functions from $X_{0}^{a b}$ are supported only on $\mathbb{A} \cup \mathbb{B}$ and the continuum test functions from $Y_{0}^{b c}$ are supported only on $\Omega_{b} \cup \Omega_{c}$. As a result, away from the bridge region the equations in the AtC blending method default to their atomistic and continuum definitions, respectively.

Due to the non-local nature of the atomistic model, the particles included in the force balance equations in $\Omega_{a} \cup \Omega_{b}$ will interact with at least some of the particles in $\Omega_{c}$. This fact is reflected in the choice of $X_{D}$ as a trial space for the atomistic part of the solution in the AtC blending method. Of course, in (6.14) we never solve for the displacements of the particles in $\Omega_{c}$, instead, whenever their displacements are needed we approximate them by the continuum displacement at the location of the particle according to the first constraint in (6.14). This requires us to assume that certain types of materials and behaviors, such as multilattice materials or phase transitions, are not present in $\Omega_{c}$.

Remark 6.1 Restricting the atomistic trial space in (6.14) to particles in $\Omega_{a} \cup \Omega_{b}$ only, will result in neglecting the forces acting on the particles in $\Omega_{a} \cup \Omega_{b}$ due to the particles in $\Omega_{c}$. This gives rise to what is known as the ghost force effect $[25,28]$. The AtC blending method (6.14) mitigates the ghost force effect in two ways. First, owing to the choice of the atomistic trial space, interactions between the particles in $\Omega_{a} \cup \Omega_{b}$ and $\Omega_{c}$ are included in the problem. Second, because the atomistic blending function $\theta_{a}$ has small values near $\Omega_{c}$, the errors caused by the use of approximate slaved atomistic displacements will be greatly reduced.

An equally important, but much less discussed issue, is the inconsistency that occurs in the continuum model at the interface between $\Omega_{a}$ and $\Omega_{b}$. Simply restricting the weak continuum equation (6.6) to $\Omega_{b} \cup \Omega_{c}$ forces the unphysical natural boundary condition $\mathcal{L}_{S}^{c}(\boldsymbol{u}) \cdot \mathbf{n}=\mathbf{0}$ along that interface. In the blended method (6.14) the adverse effects from this artificial boundary condition are greatly reduced or altogether removed thanks to the fact that $\theta_{c}=0$ on the interface between $\Omega_{a}$ and $\Omega_{b}$. 


\subsubsection{Blending functions}

With this section we begin to examine the four key ingredients of (6.14). The atomistic and continuum blending functions $\theta_{a}$ and $\theta_{c}$ are the first ingredient of a blending method. Their main purpose is to ensure that atomistic and continuum forces are blended rather than superimposed in the bridge region. In addition, judicious choice of $\theta_{a}$ can help reduce the ghost forces and errors due to slaving of the atomistic displacements in (6.14); see Remark 6.1. Likewise, with a suitable choice of $\theta_{c}$ one can avoid the imposition of an artificial natural boundary condition on the interface between $\Omega_{a}$ and $\Omega_{b}$.

It is clear that in practice, to define $\theta_{a}$ and $\theta_{c}$, it suffices to pick a single blending function $\theta$ such that $0 \leq \theta \leq 1$ in $\Omega, \theta=1$ in $\Omega_{a}$, and $\theta=0$ on $\Omega_{c}$. Then, one can set $\theta_{a}=\theta$ and $\theta_{c}=1-\theta$. A key requirement is that $\theta$ is small near the interface between $\Omega_{b}$ and $\Omega_{c}$ (so that $\theta_{a}$ is small there) and that it be close to one near the interface between $\Omega_{a}$ and $\Omega_{b}$ (so that $\theta_{c}$ is small there.) Methods for constructing the blending function $\theta$ are discussed in [4].

To achieve these desirable properties the blending functions have to be at least of class $C^{0}$. The continuum blending function $\theta_{c}$ will also be required to satisfy the following property.

\subsubsection{Assumption}

For every $\boldsymbol{v} \in Y$, we have that $\theta_{c} \boldsymbol{v} \in Y$.

\subsubsection{Enforcing the constraints}

Recall that the blended weak problem (6.14) is subject to two constraints. The first one is simply a slaving condition which postulates that atomic displacements in the continuum region are slaved to the continuum displacements. It can be trivially imposed by simply substituting the appropriate atomistic displacements in the weak equations by the associated continuum values. Thus, in what follows we shall assume that this constraint had already been enforced in the blended problem.

The second constraint is non-trivial in the sense that in the bridge region the atomistic and continuum models coexist and their displacements must be reconciled in a physically meaningful sense. As a result, the operator $\mathcal{C}$ in (6.14) can have a rather general form which may make direct imposition of this constraint more difficult. We shall discuss some specific examples of this operator after examining two possible strategies for its enforcement.

The first strategy is to use Lagrange multipliers, which leads to the mixed problem [11]: find $\{\boldsymbol{\psi}, \boldsymbol{u}\} \in X_{D} \times Y_{D}^{b c}$, and $\boldsymbol{\lambda} \in Q^{\prime}$ such that

$$
\left\{\begin{array}{cc}
\mathcal{B}_{\theta}^{a}\left(\boldsymbol{\psi}, \boldsymbol{\phi} ; \theta_{a}\right)+\mathcal{B}_{\theta}^{c}\left(\boldsymbol{u}, \boldsymbol{v} ; \theta_{c}\right)+\left\langle\left(\delta_{\boldsymbol{\psi}} \mathcal{C}(\boldsymbol{\psi}, \boldsymbol{u})\right) \boldsymbol{\phi}, \boldsymbol{\lambda}\right\rangle+\left\langle\left(\delta_{\boldsymbol{u}} \mathcal{C}(\boldsymbol{\psi}, \boldsymbol{u})\right) \boldsymbol{v}, \boldsymbol{\lambda}\right\rangle \\
=\mathcal{G}_{\theta}^{a}\left(\boldsymbol{\phi} ; \theta_{a}\right)+\mathcal{G}_{\theta}^{c}\left(\boldsymbol{v} ; \theta_{c}\right) & \forall\{\boldsymbol{\phi}, \boldsymbol{v}\} \in X_{0}^{a b} \times Y_{0}^{b c} \\
\langle\mathcal{C}(\boldsymbol{\psi}, \boldsymbol{u}), \boldsymbol{\mu}\rangle=0 & \forall \boldsymbol{\mu} \in Q^{\prime}
\end{array}\right.
$$


where $\delta_{\boldsymbol{\psi}} \mathcal{C}(\cdot, \cdot)$ and $\delta_{\boldsymbol{u}} \mathcal{C}(\cdot, \cdot)$ are the Gâteaux derivatives of $\mathcal{C}(\cdot, \cdot)$ with respect to $\boldsymbol{\psi}$ and $\boldsymbol{u}$, respectively and $Q^{\prime}$ is a suitable Lagrange multiplier space. Since $\phi \in X_{0}^{a b}$ and $\boldsymbol{v} \in Y_{0}^{b c}$ are independent of each other, (6.15) assumes the form

$$
\left\{\begin{array}{rlrl}
\mathcal{B}_{\theta}^{a}\left(\boldsymbol{\psi}, \boldsymbol{\phi} ; \theta_{a}\right)+\left\langle\left(\delta_{\boldsymbol{\psi}} \mathcal{C}(\boldsymbol{\psi}, \boldsymbol{u})\right) \boldsymbol{\phi}, \boldsymbol{\lambda}\right\rangle & =\mathcal{G}_{\theta}^{a}\left(\boldsymbol{\phi} ; \theta_{a}\right) & \forall \boldsymbol{\phi} \in X_{0}^{a b} \\
\mathcal{B}_{\theta}^{c}\left(\boldsymbol{u}, \boldsymbol{v} ; \theta_{c}\right)+\left\langle\left(\delta_{\boldsymbol{u}} \mathcal{C}(\boldsymbol{\psi}, \boldsymbol{u})\right) \boldsymbol{v}, \boldsymbol{\lambda}\right\rangle & =\mathcal{G}_{\theta}^{c}\left(\boldsymbol{v} ; \theta_{c}\right) & \forall \boldsymbol{v} \in Y_{0}^{b c} \\
\langle\mathcal{C}(\boldsymbol{\psi}, \boldsymbol{u}), \boldsymbol{\mu}\rangle & =0 & & \forall \boldsymbol{\mu} \in Q^{\prime} .
\end{array}\right.
$$

The coupling of the atomistic and continuum models in (6.16) is effected solely through the Lagrange multiplier terms in the first two equations and the constraint equation.

The second approach is to impose the constraint operator directly on the approximating spaces. Let

$$
\begin{aligned}
Z_{D}^{b} & =\left\{\boldsymbol{\psi} \in X_{D}^{b}, \boldsymbol{u} \in Y_{D}^{b} \mid \mathcal{C}(\boldsymbol{\psi}, \boldsymbol{u})=0\right\} \\
Z_{0}^{b} & =\left\{\boldsymbol{\psi} \in X_{0}^{b}, \boldsymbol{u} \in Y_{0}^{b} \mid \mathcal{C}(\boldsymbol{\psi}, \boldsymbol{u})=0\right\}
\end{aligned}
$$

where $X_{0}^{b}$ and $Y_{0}^{b}$ have the obvious definitions. Then, (6.14) assumes the following form: find $\{\boldsymbol{\psi}, \boldsymbol{u}\} \in\left(X_{D}^{a} \times Y_{D}^{c}\right) \oplus\left(X_{D}^{b} \times Y_{D}^{b}\right) \cap Z_{D}^{b}$ such that

$$
\left\{\begin{array}{l}
\mathcal{B}_{\theta}^{a}\left(\boldsymbol{\psi}, \boldsymbol{\phi} ; \theta_{a}\right)+\mathcal{B}_{\theta}^{c}\left(\boldsymbol{u}, \boldsymbol{v} ; \theta_{c}\right) \\
\quad=\mathcal{G}_{\theta}^{a}\left(\boldsymbol{\phi} ; \theta_{a}\right)+\mathcal{G}_{\theta}^{c}\left(\boldsymbol{v} ; \theta_{c}\right) \quad \forall\{\boldsymbol{\phi}, \boldsymbol{v}\} \in\left(X_{0}^{a} \times Y_{0}^{c}\right) \oplus\left(X_{0}^{b} \times Y_{0}^{b}\right) \cap Z_{0}^{b} .
\end{array}\right.
$$

The coupling between the atomistic and continuum models is now accomplished implicitly, owing to the fact that the test functions $\boldsymbol{\phi}$ and $\boldsymbol{v}$ are forced to satisfy the constraint. Among other things this implies that the split of (6.15) into (6.16) no longer possible.

Remark 6.2 The two approaches to enforce the constraints are mathematically equivalent in the sense that the solutions obtained using either one are identical. When a basis for $Z_{0}$ can be easily constructed, the advantage of (6.18) is that discretization of this problem has much fewer degrees of freedom than discretization of (6.16). This method is also more convenient from an analysis point of view because it does not require an inf-sup stability condition.

\subsubsection{Blending constraint operators}

Because the purpose of $\mathcal{C}$ is to tie together the atomistic and continuum parts of the solution in the bridge region, definition of this operator is very important for the quality of the AtC blending method. In particular, simply slaving the displacements to each other, which was appropriate in $\Omega_{c}$, may not be enough to obtain good coupling of the atomistic and continuum models. As a result, $\mathcal{C}$ can have a rather general form. In this section we focus primarily on constraint 
operators of the form

$$
\mathcal{C}_{a}(\boldsymbol{\psi})-\mathcal{C}_{c}(\boldsymbol{u})=0 \quad \text { for }\{\boldsymbol{\psi}, \boldsymbol{u}\} \in X_{D}^{b} \times Y_{D}^{b},
$$

where $\mathcal{C}_{a}(\cdot): X_{D}^{b} \rightarrow Q$ and $\mathcal{C}_{c}(\cdot): Y_{D}^{b} \rightarrow Q$ are linear operators. Two useful classes of constraint operators result from setting $\mathcal{C}_{a}$ or $\mathcal{C}_{c}$ to identity. In the first case, the constraint assumes the form

$$
\boldsymbol{\psi}=\Pi_{\mathbb{B}}(\boldsymbol{u}),
$$

where $\Pi_{\mathbb{B}}: Y_{D}^{b} \mapsto X_{D}^{b}$ is an expansion operator and $Q=X_{D}^{b}$. This operator slaves particle displacements to the continuous displacement field. As a result, atomistic degrees of freedom can be eliminated from $\Omega_{b}$. The simplest example ${ }^{3}$

$$
\boldsymbol{\psi}_{\alpha}=\boldsymbol{u}\left(\boldsymbol{x}_{\alpha}\right) \quad \forall \alpha \in \mathbb{B} \backslash(\mathbb{B} \cap \mathbb{D})
$$

embodies the physical assumption that continuous and atomistic deformation fields agree. This is precisely the case for a Cauchy-Born deformation $[18,10]$.

Setting $\mathcal{C}_{c}$ to identity gives a complementary class of constraint operators

$$
\boldsymbol{u}=\pi_{b}(\boldsymbol{\psi}),
$$

where $\pi_{b}: X_{D}^{b} \mapsto Y_{D}^{b}$ is a compression operator and $Q=Y_{D}^{b}$. In this case the continuous displacement field is slaved to particle displacements. This enables elimination of continuum degrees of freedom from $\Omega_{b}$ in a discretized AtC model.

Remark 6.3 It is important to note that using (6.20) to eliminate atomistic degrees of freedom from $\Omega_{b}$ does not delete any of the atomistic force balance equations in $\Omega_{b}$ from the AtC blending method (6.14), as is done in the quasicontinuum method [39]. Instead, using (6.20) means that $\phi$ is constrained to satisfy $\phi_{\alpha}=\boldsymbol{v}\left(\boldsymbol{x}_{\alpha}\right)$ for all $\alpha \in \mathbb{B} \backslash(\mathbb{B} \cap \mathbb{D})$, where $\boldsymbol{v}$ is the continuum test function.

More complex types of constraints, where $\mathcal{C}_{a}$ and/or $\mathcal{C}_{c}$ are not necessarily the identity operators can also be defined. We mention two examples which use subdivision $\left\{\Omega_{b, j}\right\}_{j=1}^{J}$ of $\Omega_{b}$ into $J$ nonoverlaping, covering subdomains, i.e., $\Omega_{b, j} \cap \Omega_{b, k}=\emptyset$ whenever $j \neq k$ and $\cup_{j=1}^{J} \Omega_{b, j}=\Omega_{b}$. This subdivision induces a partition of $\left\{\mathbb{B}_{j}\right\}_{j=1}^{J}$ of $\mathbb{B}$, where $\alpha \in \mathbb{B}_{j}$ whenever $\boldsymbol{x}_{\alpha} \in \Omega_{b, j}$. Let $\left|\Omega_{b, j}\right|$ and $\left|\mathbb{B}_{j}\right|$ denote the volume of $\Omega_{b, j}$ and the number of particles located in $\Omega_{b, j}$, respectively. Then,

$$
\frac{1}{\left|\Omega_{b, j}\right|} \int_{\Omega_{b, j}} \boldsymbol{u} d \boldsymbol{x}=\frac{1}{\left|\mathbb{B}_{j}\right|} \sum_{\alpha \in \mathbb{B}_{j}} \boldsymbol{\psi}_{\alpha} \quad \text { for } j=1, \ldots, J
$$

${ }^{3} \mathrm{~A}$ non-linear version of $(6.20)$; see [8], is $\left|\boldsymbol{\psi}_{\alpha}-\boldsymbol{u}\left(\boldsymbol{x}_{\alpha}\right)\right|=0 \quad \forall \alpha \in \mathbb{B} \backslash(\mathbb{B} \cap \mathbb{D})$, where $|\cdot|$ is the Euclidean norm in $\mathbb{R}^{d}$. The advantage of this operator is that it requires fewer Lagrange multipliers (one instead of $d$ per particle) than (6.20). On the other hand, (6.20) is much easier to implement. 
defines a set of constraints that is less stringent than (6.20). The integral can be approximated by a simple average to obtain another version of this operator:

$$
\sum_{\alpha \in \mathbb{B}_{j}} \boldsymbol{u}\left(\boldsymbol{x}_{\alpha}\right)=\sum_{\alpha \in \mathbb{B}_{j}} \boldsymbol{\psi}_{\alpha} \quad \text { for } j=1, \ldots, J .
$$

In either case, we have that $Q=\mathbb{R}^{J d}$.

Constraints such as (6.22) involve linear combinations of displacements and are difficult to enforce on the trial spaces. In this case, the Lagrange multiplier approach is more useful. For the constraint equations (6.22), one defines the Lagrange multipliers to be piecewise constant functions with respect to the subdivision $\left\{\Omega_{b, j}\right\}_{j=1}^{J}$ of $\Omega_{b}$.

\subsubsection{Consistency and patch tests}

We conclude with formal definitions of consistency and patch tests introduced in $[5]$.

\subsubsection{Definition [Consistency test problem]}

The set $\left\{\boldsymbol{\chi}, \boldsymbol{\psi}^{\mathbb{D}} ; \boldsymbol{f}, \boldsymbol{u}^{\partial \Omega}\right\}$ is called a consistency test problem if the solutions $\widetilde{\boldsymbol{\psi}}$ and $\widetilde{\boldsymbol{u}}$ of the global problems (6.2) and (6.6), respectively, ${ }^{4}$ are such that $\mathcal{C}(\widetilde{\boldsymbol{\psi}}, \widetilde{\boldsymbol{u}})=0$ holds on $\Omega$.

\subsubsection{Definition [Patch test problem]}

A consistency test problem is called patch test problem if the continuous component $\widetilde{\boldsymbol{u}}$ of $(\widetilde{\boldsymbol{\psi}}, \widetilde{\boldsymbol{u}})$ is such that $\mathcal{L}_{S}^{c}(\widetilde{\boldsymbol{u}})$ is constant, i.e., $\widetilde{\boldsymbol{u}}$ is a constant stress solution.

The following definitions formalize the notion of passing a patch test and a definition of consistency for an AtC coupling method.

\subsubsection{Definition [Passing a patch test problem]}

Assume that $\left\{\boldsymbol{\chi}, \boldsymbol{\psi}^{\mathbb{D}} ; \boldsymbol{f}, \boldsymbol{u}^{\partial \Omega}\right\}$ is a patch test problem with solution $(\widetilde{\boldsymbol{\psi}}, \widetilde{\boldsymbol{u}})$. An AtC coupling method passes a patch test if $(\widetilde{\boldsymbol{\psi}}, \widetilde{\boldsymbol{u}})$ satisfies the AtC coupled problem (6.16) or (6.18).

\subsubsection{Definition [AtC consistency]}

An AtC coupling method is consistent if, for any consistency test problem, the pair $(\widetilde{\boldsymbol{\psi}}, \widetilde{\boldsymbol{u}})$ satisfies the coupled AtC system.

Atomistic problems with Cauchy-Born solutions (see $[18,10])$ are a physical example of consistency test problems. From the previous definitions, one can easily infer that consistency implies passage of the patch test problem. However, the converse statement is not true.

\subsubsection{Blended atomistic and continuum functionals}

Assuming that blending functions, constraint operators and a method for their enforcement have already been chosen, all that remains to be done to obtain an

${ }^{4}$ Recall that $\left\{\boldsymbol{\chi}, \boldsymbol{\psi}^{\mathbb{D}} ; \boldsymbol{f}, \boldsymbol{u}^{\partial \Omega}\right\}$ provides the data for these two problems. 
AtC method is to define the blended functionals $\mathcal{B}_{\theta}^{c}, \mathcal{B}_{\theta}^{a}, \mathcal{G}_{\theta}^{c}$, and $\mathcal{G}_{\theta}^{a}$ appearing in $(6.14)$.

There are two possible ways to obtain these functionals from the original atomistic and continuum functionals (6.3) and (6.5). The first one is to use $\mathcal{B}^{a}(\boldsymbol{\psi}, \boldsymbol{\phi})$ and/or $\mathcal{B}^{c}(\boldsymbol{u}, \boldsymbol{v})$ directly without changing their definitions. We call this approach external blending because it preserves the internal definitions of force balance from (6.3) and (6.5). The second possibility is to define $\mathcal{B}_{\theta}^{c}$ and $\mathcal{B}_{\theta}^{a}$ by modifying $\mathcal{B}^{a}(\boldsymbol{\psi}, \boldsymbol{\phi})$ and $\mathcal{B}^{c}(\boldsymbol{u}, \boldsymbol{v})$ over $\Omega_{b}$. We call this approach internal blending because it modifies the internal definition of the force balance in (6.3) and (6.5).

For the atomistic blended functional these choices are

$$
\mathcal{B}_{\theta}^{a}\left(\boldsymbol{\psi}, \boldsymbol{\phi} ; \theta_{a}\right)= \begin{cases}\mathcal{B}^{a}\left(\boldsymbol{\psi}, \boldsymbol{\Theta}_{a} \boldsymbol{\phi}\right)=\left(\mathcal{L}^{a}(\boldsymbol{\psi}), \boldsymbol{\Theta}_{\boldsymbol{a}} \boldsymbol{\phi}\right) & \Longleftarrow \text { external } \\ \text { or } & \\ \left(\mathcal{L}_{\theta}^{a}\left(\boldsymbol{\psi} ; \theta_{a}\right), \boldsymbol{\phi}\right) & \Longleftarrow \text { internal }\end{cases}
$$

for all $\boldsymbol{\psi} \in X_{D}$ and $\phi \in X_{0}^{a b}$, where $\boldsymbol{\Theta}_{\boldsymbol{a}}$ is a diagonal weighting matrix whose diagonal values are equal to $\theta_{a}$ evaluated at the corresponding particle positions:

$$
\left(\boldsymbol{\Theta}_{a}\right)_{\alpha \beta}^{i j}=\delta_{i j} \delta_{\alpha \beta} \theta_{a}\left(\boldsymbol{x}_{\alpha}\right), \quad \text { for } i, j=1, \ldots, d, \alpha, \beta \in \mathbb{P} \text {. }
$$

For examples of how $\mathcal{L}_{\theta}^{a}\left(\boldsymbol{\psi} ; \theta_{a}\right)$ may be defined we refer to [4].

For the continuum blended functional the choices are

$$
\mathcal{B}_{\theta}^{c}\left(\boldsymbol{u}, \boldsymbol{v} ; \theta_{c}\right)= \begin{cases}\mathcal{B}^{c}\left(\boldsymbol{u}, \theta_{c} \boldsymbol{v}\right)=\int_{\Omega_{b} \cup \Omega_{c}} \mathcal{L}_{S}^{c}(\boldsymbol{u}): \mathcal{L}_{E}^{c}\left(\theta_{c} \boldsymbol{v}\right) d \boldsymbol{x} & \Longleftarrow \text { external } \\ \int_{\Omega_{b} \cup \Omega_{c}} \theta_{c} \mathcal{L}_{S}^{c}(\boldsymbol{u}): \mathcal{L}_{E}^{c}(\boldsymbol{v}) d \boldsymbol{x} & \Longleftarrow \text { internal }\end{cases}
$$

for all $\boldsymbol{u} \in Y_{D}^{b c}$ and $\boldsymbol{v} \in Y_{0}^{b c}$.

Remark 6.4 The difference between internal and external blending can be further appreciated by examining the strong forms of the differential operators in (6.24). Suppose that $\mathcal{L}^{c}=-\Delta$ is the Laplace operator. The blended versions of $\mathcal{L}^{c}$ are

$$
\mathcal{B}_{\theta}^{c}\left(u, v ; \theta_{c}\right)= \begin{cases}\int_{\Omega_{b} \cup \Omega_{c}} \nabla(u) \cdot \nabla\left(\theta_{c} v\right) d \boldsymbol{x} & \Longleftarrow \text { external } \\ \int_{\Omega_{b} \cup \Omega_{c}} \theta_{c} \nabla(u) \cdot \nabla(v) d \boldsymbol{x} & \Longleftarrow \text { internal }\end{cases}
$$

The strong forms of the externally and internally blended operators are

$$
\mathcal{L}_{E}^{c}=-\theta_{c} \Delta \quad \text { and } \quad \mathcal{L}_{I}^{c}=-\nabla \cdot \theta_{c} \nabla,
$$

respectively. We see that external blending only scales $\mathcal{L}^{c}$ without changing its definition. In contrast, internal blending modifies $\mathcal{L}^{c}$ in the blend region which effectively changes the response of the continuum material there. 
For either one of the choices in (6.23) and (6.24), the blended linear data functionals appearing in (6.14) are defined ${ }^{5}$ as

$$
\mathcal{G}_{\theta}^{a}\left(\phi ; \theta_{a}\right)=\mathcal{G}^{a}\left(\Theta_{a} \phi\right)=-\left(\chi, \Theta_{a} \phi\right)=-\left(\Theta_{a} \chi, \phi\right) \quad \forall \phi \in X_{0}^{a b}
$$

and

$$
\mathcal{G}_{\theta}^{c}\left(\boldsymbol{v} ; \theta_{c}\right)=\mathcal{G}^{c}\left(\theta_{c} \boldsymbol{v}\right)=\left\langle\boldsymbol{f}, \theta_{c} \boldsymbol{v}\right\rangle=\left\langle\theta_{c} \boldsymbol{f}, \boldsymbol{v}\right\rangle=\int_{\Omega_{b} \cup \Omega_{c}} \theta_{c} \boldsymbol{f} \cdot \boldsymbol{v} d \boldsymbol{x} \quad \forall \boldsymbol{v} \in Y_{0}^{b c},
$$

respectively.

\subsubsection{Taxonomy of AtC blending methods}

Because external and internal blendings can be applied independently to the continuum and atomistic problems there are four possible ways to define the blended AtC functional $\mathcal{B}^{a}\left(\boldsymbol{\psi}, \boldsymbol{\phi} ; \theta_{a}\right)+\mathcal{B}_{\theta}^{c}\left(\boldsymbol{u}, \boldsymbol{v} ; \theta_{c}\right)$. These choices are summarized in Table 6.1.

Below we review each one of the four types ot AtC blending methods and comment on their properties.

\subsubsection{Methods of type I}

For external atomistic and internal continuum blending the abstract AtC method (6.14) assumes the form: find $\{\boldsymbol{\psi}, \boldsymbol{u}\} \in X_{D} \times Y_{D}^{b c}$ such that

$$
\left\{\begin{array}{c}
\left(\mathcal{L}^{a}(\boldsymbol{\psi}), \boldsymbol{\Theta}_{\boldsymbol{a}} \boldsymbol{\phi}\right)+\int_{\Omega_{b} \cup \Omega_{c}} \theta_{c} \mathcal{L}_{S}^{c}(\boldsymbol{u}): \mathcal{L}_{E}^{c}(\boldsymbol{v}) d \boldsymbol{x} \\
=-\left(\boldsymbol{\chi}, \boldsymbol{\Theta}_{\boldsymbol{a}} \boldsymbol{\phi}\right)+\int_{\Omega_{b} \cup \Omega_{c}} \theta_{c} \boldsymbol{f} \cdot \boldsymbol{v} d \boldsymbol{x} \quad \forall\{\boldsymbol{\phi}, \boldsymbol{v}\} \in X_{0}^{a b} \times Y_{0}^{b c} \\
\mathcal{C}(\boldsymbol{\psi}, \boldsymbol{u})=0 \quad \forall\{\boldsymbol{\psi}, \boldsymbol{u}\} \in X_{D}^{b} \times Y_{D}^{b}
\end{array}\right.
$$

For this method, we have the following result [5].

Table 6.1. Force-based AtC blending methods classified by blending types

\begin{tabular}{lll}
\hline & \multicolumn{2}{c}{ Type of the blending } \\
\cline { 2 - 3 } Type of the method & Atomistic model & Continuum model \\
\hline I & external & internal \\
II & internal & internal \\
III & external & external \\
IV & internal & external \\
\hline
\end{tabular}

\footnotetext{
${ }^{5}$ We can restrict the integrals in (6.24) and (6.27) to $\Omega_{b} \cup \Omega_{c}$ because, by the definition of the test space $Y_{0}^{b c}$, the continuum test function $\boldsymbol{v}$ is supported only within that subregion.
} 


\subsubsection{Theorem}

Methods of Type I are inconsistent and do not pass the patch test.

This conclusion also extends to discretizations of Type I methods where the continuum part is approximated by, e.g., finite elements.

\subsubsection{Methods of type II}

For internal atomistic and continuum blending the abstract AtC method (6.14) assumes the form: find $\{\boldsymbol{\psi}, \boldsymbol{u}\} \in X_{D} \times Y_{D}^{b c}$ such that

$$
\left\{\begin{aligned}
&\left(\mathcal{L}_{\theta}^{a}\left(\boldsymbol{\psi} ; \theta_{a}\right), \boldsymbol{\phi}\right)+\int_{\Omega_{b} \cup \Omega_{c}} \theta_{c} \mathcal{L}_{S}^{c}(\boldsymbol{u}): \mathcal{L}_{E}^{c}(\boldsymbol{v}) d \boldsymbol{x} \\
&=-\left(\boldsymbol{\chi}, \boldsymbol{\Theta}_{a} \boldsymbol{\phi}\right)+\int_{\Omega_{b} \cup \Omega_{c}} \theta_{c} \boldsymbol{f} \cdot \boldsymbol{v} d \boldsymbol{x} \quad \forall\{\boldsymbol{\phi}, \boldsymbol{v}\} \in X_{0}^{a b} \times Y_{0}^{b c} \\
& \mathcal{C}(\boldsymbol{\psi}, \boldsymbol{u})=0 \quad \forall\{\boldsymbol{\psi}, \boldsymbol{u}\} \in X_{D}^{b} \times Y_{D}^{b}
\end{aligned}\right.
$$

Consistency of Type II methods depends on the definition of $\mathcal{L}_{\theta}^{a}\left(\cdot ; \theta_{a}\right)$. The following theorem [5] gives an abstract consistency condition for this operator.

\subsubsection{Theorem}

Under Assumption 6.3.3, a sufficient condition for Type II methods to be consistent is that for any consistency test problem solution $\{\widetilde{\boldsymbol{\psi}}, \widetilde{\boldsymbol{u}}\}$ the identity

$$
\begin{aligned}
& \left(\mathcal{L}_{\theta}^{a}\left(\widetilde{\boldsymbol{\psi}} ; \theta_{a}\right), \boldsymbol{\phi}\right)-\left(\mathcal{L}^{a}(\widetilde{\boldsymbol{\psi}}), \boldsymbol{\Theta}_{a} \boldsymbol{\phi}\right) \\
& \quad=-\int_{\Omega_{b} \cup \Omega_{c}}\left(\theta_{c} \mathcal{L}_{S}^{c}(\widetilde{\boldsymbol{u}}): \mathcal{L}_{E}^{c}(\boldsymbol{v})-\mathcal{L}_{S}^{c}(\widetilde{\boldsymbol{u}}): \mathcal{L}_{E}^{c}\left(\theta_{c} \boldsymbol{v}\right)\right) d \boldsymbol{x}
\end{aligned}
$$

holds for all $\{\boldsymbol{\phi}, \boldsymbol{v}\} \in X_{0}^{a b} \times Y_{0}^{b c}$. Type II methods pass the patch test if (6.30) is satisfied for patch test solutions.

Remark 6.5 It is not hard to see that the reason Type I methods fail to be consistent is that for any solution of a consistency test problem the atomistic terms in (6.28) vanish but the continuum terms do not. By using internal atomistic and continuum blending, Type II methods make it possible to "cancel" consistency errors by using a suitably defined $\mathcal{L}_{\theta}^{a}$. Indeed, the right hand side in (6.30) and the term $\left(\mathcal{L}^{a}(\widetilde{\boldsymbol{\psi}}), \boldsymbol{\Theta}_{a} \boldsymbol{\phi}\right)$ on the left hand side are completely defined once $\theta_{c}$ and $\theta_{a}$ have been selected. Then, one can choose $\mathcal{L}_{\theta}^{a}$ so that (6.30) holds.

In [4], a specific choice for $\mathcal{L}_{\theta}^{a}\left(\cdot ; \theta_{a}\right)$ is defined that satisfies $(6.30)$ for a particular set of one-dimensional patch test problems. The choice can be mechanically justified as a blended force balance.

It is worth pointing out that among the four methods discusssed here, Type II methods are the only one that can preserve the symmetry of the underlying atomistic and continuum problems. This has a positive effect on their stability and leads to symmetric linear systems that are easier to solve than the nonsymmetric linear systems generated by the other three methods. 
6.3.13.1 Methods of type III

For external atomistic and continuum blending the abstract AtC method (6.14) assumes the form: find $\{\boldsymbol{\psi}, \boldsymbol{u}\} \in X_{D} \times Y_{D}^{b c}$ such that

$$
\left\{\begin{array}{c}
\left(\mathcal{L}^{a}(\boldsymbol{\psi}), \boldsymbol{\Theta}_{a} \boldsymbol{\phi}\right)+\int_{\Omega_{b} \cup \Omega_{c}} \mathcal{L}_{S}^{c}(\boldsymbol{u}): \mathcal{L}_{E}^{c}\left(\theta_{c} \boldsymbol{v}\right) d \boldsymbol{x} \\
=-\left(\boldsymbol{\chi}, \boldsymbol{\Theta}_{\boldsymbol{a}} \boldsymbol{\phi}\right)+\int_{\Omega_{b} \cup \Omega_{c}} \theta_{c} \boldsymbol{f} \cdot \boldsymbol{v} d \boldsymbol{x} \quad \forall\{\boldsymbol{\phi}, \boldsymbol{v}\} \in X_{0}^{a b} \times Y_{0}^{b c} \\
\mathcal{C}(\boldsymbol{\psi}, \boldsymbol{u})=0 \quad \forall\{\boldsymbol{\psi}, \boldsymbol{u}\} \in X_{D}^{b} \times Y_{D}^{b}
\end{array}\right.
$$

Type III methods can be interpreted as residual blending methods because they merge the continuum residual $\mathcal{L}^{c} \boldsymbol{u}-\boldsymbol{f}$ with the atomistic residual $\mathcal{L}^{a}(\boldsymbol{\psi})-\boldsymbol{\chi}$. This fact endows Type III methods with an attractive feature that is not shared by the other three AtC blending methods: for a consistency problem solution, the atomistic and continuum terms in (6.29) separately cancel out and so, this method is intrinsically consistent. This observation is formalized in the following theorem [5].

\subsubsection{Theorem}

Under Assumption 6.3.3, Type III methods are consistent and pass the patch test.

\subsubsection{Methods of type IV}

For internal atomistic and external continuum blending the abstract AtC method (6.14) assumes the form: find $\{\boldsymbol{\psi}, \boldsymbol{u}\} \in X_{D} \times Y_{D}^{b c}$ such that

$$
\left\{\begin{aligned}
&\left(\mathcal{L}_{\theta}^{a}\left(\boldsymbol{\psi} ; \theta_{a}\right), \boldsymbol{\phi}\right)+\int_{\Omega_{b} \cup \Omega_{c}} \theta_{c} \mathcal{L}_{S}^{c}(\boldsymbol{u}): \mathcal{L}_{E}^{c}(\boldsymbol{v}) d \boldsymbol{x} \\
&=-\left(\boldsymbol{\chi}, \boldsymbol{\Theta}_{a} \boldsymbol{\phi}\right)+\int_{\Omega_{b} \cup \Omega_{c}} \theta_{c} \boldsymbol{f} \cdot \boldsymbol{v} d \boldsymbol{x} \quad \forall\{\boldsymbol{\phi}, \boldsymbol{v}\} \in X_{0}^{a b} \times Y_{0}^{b c} \\
& \mathcal{C}(\boldsymbol{\psi}, \boldsymbol{u})=0 \quad \forall\{\boldsymbol{\psi}, \boldsymbol{u}\} \in X_{D}^{b} \times Y_{D}^{b}
\end{aligned}\right.
$$

The types of the atomistic and the continuum blending in Type IV methods are reversed with respect to Type I methods. As a result, these methods can be thought of as "dual" to Type I. The duality of the two classes is further underscored by the fact that in Type IV methods the continuous terms cancel for any consistency problem solution but the atomistic terms do not; exactly the opposite was true for Type I methods. Clearly, Type IV methods are also inconsistent.

\subsubsection{Summary and comparison of force-based AtC blending methods}

Consistency properties of the four types of AtC blending methods are compared and contrasted in Table 6.2.

AtC blending methods of Types I and II have appeared previously. For example, according to our classification scheme, the AtC blending method described in [8] is of Type I; see equations (10) and (11) of that paper. An example of a 
Table 6.2. Atomistic and continuous contributions in AtC methods at a consistency problem solution $\{\widetilde{\boldsymbol{\psi}}, \widetilde{\boldsymbol{u}}\}$

\begin{tabular}{cccc}
\hline Type of the method & $\mathcal{B}_{\theta}^{a}(\widetilde{\boldsymbol{\psi}}, \boldsymbol{\phi})-\mathcal{G}_{\theta}^{a}(\boldsymbol{\phi})$ & $\mathcal{B}_{\theta}^{c}(\widetilde{\boldsymbol{u}}, \boldsymbol{v})-\mathcal{G}_{\theta}^{c}(\boldsymbol{v})$ & Consistent? \\
\hline I & $=0$ & $\neq 0$ & No \\
II & $\neq 0$ & $\neq 0$ & Depends on $\mathcal{L}_{\theta_{a}}^{a}$ \\
III & $=0$ & $=0$ & Yes \\
IV & $\neq 0$ & $=0$ & No \\
\hline
\end{tabular}

Type II method can be found in $[4,20]$. We also remark that Methods of types I, III, and IV do not satisfy Newton's third law over the blend region, and so these methods do not lead to a symmetric formulation.

We also contrast AtC blending with the quasicontinuum method [39]. In a local quasicontinuum method, the Cauchy-Born hypothesis [10] is used to eliminate degrees of freedom in a particle model, lessening the computational complexity. The local quasicontinuum approximation has no direct relation to blending. In certain circumstances, the local/nonlocal interface arising in the quasicontinuum method can be viewed as the blending approach of Type II methods with a $d-1$ dimensional interface; see [14]. Furthermore, the forces in the quasicontinuum method are derived from a global energy functional and obey Newton's third law (or equivalently, the conservation of linear momentum).

\subsection{Energy-based blending}

Obviously we can always tie together energy based atomistic and continuum models by blending weak forms of their associated first-order optimality systems. This optimize and then blend approach reduces formulation of AtC methods for energy-based models to the force-based setting from the last section. In this section we shall consider a bona fide energy-based blending that can be described as blend and then optimize approach.

\subsubsection{An abstract AtC blending method}

In the blend and then optimize approach the first two key blending ingredients, i.e., the blending functions and the constraint operator $\mathcal{C}$, are shared with the force-based approach but the last two are different and are derived from the atomistic (6.7) and continuum (6.10) potential energy functionals. Specifically, instead of $\mathcal{B}_{\theta}^{a}\left(\cdot, \cdot ; \theta_{a}\right)$ and $\mathcal{B}_{\theta}^{c}\left(\cdot, \cdot ; \theta_{c}\right)$ we now consider:

- a blended atomistic potential energy functional

$$
\mathcal{E}_{\theta}^{a}\left(\boldsymbol{\psi} ; \theta_{a}\right)=\sum_{\alpha \in \mathbb{A} \cup \mathbb{B}}\left(W_{\alpha ; \theta_{a}}^{a}(\boldsymbol{\psi})+\boldsymbol{\chi}_{\alpha ; \theta_{a}} \cdot \boldsymbol{\psi}_{\alpha}\right) \text { such that } \mathcal{E}_{\theta}^{a}(\boldsymbol{\psi} ; 1)=\mathcal{E}^{a}(\boldsymbol{\psi})
$$


- a blended continuum potential energy functional

$$
\mathcal{E}_{\theta}^{c}\left(\boldsymbol{u} ; \theta_{c}\right)=\int_{\Omega_{b} \cup \Omega_{c}}\left(W_{\theta_{c}}(\boldsymbol{u})+\mathbf{f}_{\theta_{c}} \cdot \boldsymbol{u}\right) d \Omega \text { such that } \mathcal{E}_{\theta}^{c}(\boldsymbol{u} ; 1)=\mathcal{E}^{c}(\boldsymbol{u}) .
$$

Using these definitions, an abstract, energy-based AtC blending method can be stated as the following constrained optimization problem (compare with $(6.14))$ :

$$
\left\{\begin{array}{c}
\operatorname{minimize} \mathcal{E}_{\theta}\left(\boldsymbol{\psi}, \boldsymbol{u} ; \theta_{a}, \theta_{c}\right)=\mathcal{E}_{\theta}^{a}\left(\boldsymbol{\psi} ; \theta_{a}\right)+\mathcal{E}_{\theta}^{c}\left(\boldsymbol{u} ; \theta_{c}\right) \\
\text { subject to } \\
\boldsymbol{\psi}_{\alpha}=\boldsymbol{u}\left(\boldsymbol{x}_{\alpha}\right) \forall \alpha \in \mathbb{C} \backslash(\mathbb{C} \cap \mathbb{D}) \text { and } \mathcal{C}(\boldsymbol{\psi}, \boldsymbol{u})=0 \forall\{\boldsymbol{\psi}, \boldsymbol{u}\} \in X_{D}^{b} \times Y_{D}^{b}
\end{array}\right.
$$

The blended atomistic-continuum potential energy $\mathcal{E}_{\theta}\left(\boldsymbol{\psi}, \boldsymbol{u} ; \theta_{a}, \theta_{c}\right)$ describes a hybrid model that is neither continuous nor atomistic. However, owing to the definition of blended atomistic and continuum energies, $\mathcal{E}_{\theta}$ has the following additive property:

$$
\begin{aligned}
& \mathcal{E}_{\theta}\left(\boldsymbol{u}, \boldsymbol{\psi} ; \theta_{a}, \theta_{c}\right) \\
&=\sum_{\alpha \in \mathbb{A}}\left(W_{\alpha}^{a}(\boldsymbol{\psi})+\boldsymbol{\chi}_{\alpha} \cdot \boldsymbol{\psi}_{\alpha}\right) \rightarrow \text { atomistic energy in atomistic region } \\
& \quad+\int_{\Omega_{c}}\left(W^{c}(\boldsymbol{u})+\mathbf{f} \cdot \boldsymbol{u}\right) d \Omega \quad \rightarrow \text { continuum energy in continuum region } \\
& \quad+\sum_{\alpha \in \mathbb{B}}\left(W_{\alpha ; \theta_{a}}^{c}(\boldsymbol{\psi})+\boldsymbol{\chi}_{\alpha ; \theta_{a}} \cdot \boldsymbol{\psi}_{\alpha}\right) \rightarrow \text { atomistic energy in bridge region } \\
& \quad+\int_{\Omega_{b}}\left(W_{\theta_{c}}^{c}(\boldsymbol{u})+\mathbf{f}_{\theta_{c}} \cdot \boldsymbol{u}\right) d \Omega \quad \rightarrow \text { continuum energy in bridge region }
\end{aligned}
$$

The first and the second terms are simply the atomistic and continuum potential energies for the atomistic and continuum subdomains, respectively. The third and fourth terms together represent the blended energy in the bridge region. Different definitions of $W_{\theta_{c}}^{c}, W_{\alpha ; \theta_{a}}^{a}$, etc., will result in different blending methods.

\subsubsection{Enforcing the constraints}

Similarly to the weak force-based weak problem (6.14) the blended optimization problem (6.33) is subject to two constraints. The first one is the usual slaving condition which ties atomistic displacements in $\Omega_{c}$ to the continuum displacements. As in the force-based case, this constraint can be trivially imposed by simply substituting the appropriate atomistic displacements in the energy functional by the associated continuum values. Thus, in what follows we shall assume that this constraint had already been enforced in (6.33). 
The second, blending, constraint in (6.33) serves the same purpose as in the force-based blending: its role is to tie together the atomistic and continuum solutions over the bridge region. Examples of such operators were given in Section 6.3.4.1; the same blending constraints can also be applied in energy-based methods. To enforce these constraints we again have a choice of two different strategies.

The first one uses Lagrange multipliers to replace the constrained optimization problem (6.33) by the unconstrained problem of finding the saddle-point $\{\{\boldsymbol{\psi}, \boldsymbol{u}\}, \boldsymbol{\lambda}\} \in\left\{X_{D} \times Y_{D}^{b c}\right\} \times Q^{\prime}$ of the Lagrangian functional

$$
\mathcal{L}\left(\boldsymbol{u}, \boldsymbol{\psi}, \vec{\lambda} ; \theta_{a}, \theta_{c}\right)=\mathcal{E}_{\theta}\left(\boldsymbol{u}, \boldsymbol{\psi} ; \theta_{a}, \theta_{c}\right)+\langle\mathcal{C}(\boldsymbol{u}, \boldsymbol{\psi}), \boldsymbol{\lambda}\rangle
$$

According to the Lagrange multiplier rule, we can now take independent variations in each of $\boldsymbol{\psi}, \boldsymbol{u}$, and $\boldsymbol{\lambda}$ to obtain the optimality system

$$
\left\{\begin{array}{l}
\delta_{\boldsymbol{\psi}} \mathcal{E}_{\theta}\left(\boldsymbol{u}, \boldsymbol{\psi} ; \theta_{a}, \theta_{c}\right)+\left\langle\delta_{\boldsymbol{\psi}} \mathcal{C}(\boldsymbol{\psi}, \boldsymbol{u}), \boldsymbol{\lambda}\right\rangle=0 \\
\delta_{\boldsymbol{u}} \mathcal{E}_{\theta}\left(\boldsymbol{u}, \boldsymbol{\psi} ; \theta_{a}, \theta_{c}\right)+\left\langle\delta_{\boldsymbol{u}} \mathcal{C}(\boldsymbol{\psi}, \boldsymbol{u}), \boldsymbol{\lambda}\right\rangle=0 \\
\mathcal{C}(\boldsymbol{u}, \boldsymbol{\psi})=0
\end{array}\right.
$$

Note that since $\mathcal{E}_{\theta}\left(\boldsymbol{u}, \boldsymbol{\psi} ; \theta_{a}, \theta_{c}\right)$ is a sum of functionals of $\boldsymbol{u}$ only and of $\boldsymbol{\psi}$ only, that $\delta_{\boldsymbol{u}} \mathcal{E}_{\theta}$ depends only on $\boldsymbol{u}$ and $\delta_{\boldsymbol{\psi}} \mathcal{E}_{\theta}$ depends only on $\boldsymbol{\psi}$. Thus, coupling is effected solely by the Lagrange multiplier terms which mirrors the force-based setting. In particular, the structure of (6.34) closely resembles that of (6.16).

The second strategy to enforce the constraints, used in force-based blending methods, is also applicable to energy-based methods. We remind that in this approach the constraints are imposed on the solution spaces, which transforms (6.33) into the following unconstrained minimization problem: find ${ }^{6}\{\boldsymbol{\psi}, \boldsymbol{u}\} \in$ $\left(X_{D}^{a} \times Y_{D}^{c}\right) \oplus\left(X_{D}^{b} \times Y_{D}^{b}\right) \cap Z_{D}^{b}$ such that

$$
\mathcal{E}_{\theta}\left(\boldsymbol{u}, \boldsymbol{\psi} ; \theta_{a}, \theta_{c}\right) \leq \mathcal{E}_{\theta}\left(\boldsymbol{v}, \boldsymbol{\phi} ; \theta_{a}, \theta_{c}\right) \quad \forall\{\boldsymbol{\phi}, \boldsymbol{v}\} \in\left(X_{0}^{a} \times Y_{0}^{c}\right) \oplus\left(X_{0}^{b} \times Y_{0}^{b}\right) \cap Z_{0}^{b} .
$$

Problem (6.35) is the counterpart of the blended weak problem (6.18).

\subsubsection{Taxonomy of AtC blending methods}

In many ways formulation of energy-based AtC blending methods closely follows that of force-based methods, and gives rise to problems with like structures. In Section 6.3.11 we identified four different types of force-based AtC blending methods. Roughly speaking, these four types corresponded to the number of possible ways to allocate the blending functions between test and trial functions in (6.14). We called the blending "external" when the blending function was assigned to the test function and "internal" otherwise.

\footnotetext{
${ }^{6}$ The spaces $Z_{D}^{b}$ and $Z_{0}^{b}$ were defined in (6.17).
} 
In energy-based blending there are no test and trial functions but analogues of internal and external blending still exists. Since external blending is not supposed to change the mechanical definition of the potential energy, in the present case it corresponds to a weighting of the energy functional. For the atomistic potential energy we thus have the choice of

$$
\mathcal{E}_{\theta}^{a}\left(\boldsymbol{\psi} ; \theta_{a}\right)=\left\{\begin{aligned}
\sum_{\alpha \in \mathbb{A} \cup \mathbb{B}} \theta_{a}\left(\boldsymbol{x}_{\alpha}\right)\left(W_{\alpha}^{a}(\boldsymbol{\psi})+\boldsymbol{\chi}_{\alpha} \cdot \boldsymbol{\psi}_{\alpha}\right) & \Longleftarrow \text { oxternal } \\
\sum_{\alpha \in \mathbb{A} \cup \mathbb{B}}\left(W_{\alpha ; \theta_{a}}^{c}(\boldsymbol{\psi})+\boldsymbol{\chi}_{\alpha ; \theta_{a}} \cdot \boldsymbol{\psi}_{\alpha}\right) & \Longleftarrow \text { internal }
\end{aligned}\right.
$$

Similarly, for the continuum blended potential energy the choices are

$$
\mathcal{E}_{\theta}^{c}\left(\boldsymbol{u} ; \theta_{c}\right)= \begin{cases}\int_{\Omega_{b} \cup \Omega_{c}} \theta_{c}(\boldsymbol{x})\left(W^{c}(\boldsymbol{u})+\mathbf{f} \cdot \boldsymbol{u}\right) d \Omega & \Longleftarrow \text { external } \\ \int_{\Omega_{b} \cup \Omega_{c}}\left(W_{\theta_{c}}^{c}(\boldsymbol{u})+\mathbf{f}_{\theta_{c}} \cdot \boldsymbol{u}\right) d \Omega & \Longleftarrow \text { internal }\end{cases}
$$

It follows that formally, energy-based blending can also result in four different types of AtC blending methods. Perhaps the two most important cases are the analogues of Type II and Type III methods, in which the type of the blending is the same for the atomistic and continuum energies. The overlapping domain decomposition coupling method in [8] is an example of a Type II blending method.

It is not clear whether or not analogues of Type I and Type IV methods would be useful at all in the energy-based coupling context. One concern is that by mixing different blending strategies it may be very difficult to ensure that the energy in the blend regions is not over or under-counted.

\subsection{Generalized continua}

The intrinsic incompatibility of coupling mechanical models with local/nonlocal force interaction suggests that we consider generalizations of classical continuum mechanics as described in $[7,13,19,35,36,37]$, or continuum realizations of molecular dynamics $[1,42,12,43,30]$. We refer to these classes of methods as generalized continua. They are motivated, in large part, by introducing a lengthscale (absent in classical elasticity) by augmenting the displacement field with supplementary fields (e.g., rotations) that provide information about fine-scale kinematics, by using higher-order gradients of the displacement field, by averaging local strains and/or stresses, or by introducing a notion of a field into molecular dynamics. We also mention the papers $[15,23]$ where variational principles for the generalized continua are described useful for finite element based discretizations, and [21] where the classical theory is augmented with internal bonds. 
The blending methods reviewed in this paper may also be applied for use with generalized continua, and require modification to (6.4), and the equations that follow. In particular, $\mathcal{L}^{c}(\boldsymbol{u}), \mathcal{L}_{S}^{c}(\cdot)$ and $\mathcal{L}_{E}^{c}(\cdot)$ may not correspond to a differential operator, and a continuum notion of the force operator $\mathcal{L}^{a}$ introduced in (6.1) may need modification.

A generalized continua can fulfill two roles within a blending method. The first is to replace the classical elastic theory, the second is as an intermediatory between molecular dynamics and classical elasticity. The second approach becomes viable if there is theoretical justification linking the generalized continua with classical elasticity, especially if the force interaction model is nonlocal.

For instance, the recent paper [38] explains that if the underlying deformation is sufficiently smooth, then peridynamics [36, 37] is asymptotically equivalent [38] to classical elasticity as the length-scale decreases, and has a symbiotic relationship with molecular dynamics. As a result, a discretized peridynamic model can be implemented using an off-the-shelf molecular dynamic code as described in [32]. Future work blends molecular dynamics and peridynamics to enable multiscale materials modeling.

\subsection{Conclusions}

In this paper we reviewed a novel mathematical framework for encoding and classifying force or energy-based AtC blending methods. In particular, we identified four key ingredients of such methods and two possible blending types that can be applied in the force-based or energy-based settings. We also stated formal definitions of consistency and patch tests for AtC blending methods, and explained how the ghost force effects and unphysical interface boundary conditions are mitigated in such methods.

Based on the type of blending applied to the atomistic and continuum models, AtC blending methods can be divided into four different categories. Only one of these categories, Type II methods, leads to AtC formulations that are simultaneously consistent, symmetric and do not violate Newton's third law over the bridge region.

Equally important for the AtC blending methods is the choice of the blending constraint operator whose purpose is to reconcile atomistic and continuum displacements in the bridge region so as to impose a suitable notion of "continuity" in the coupled solution. The choice of how to enforce these constraints does not modify the final result, but has important implications for the implementation of AtC methods. We have considered two different choices: classical Lagrange multipliers, and restricted AtC spaces whose elements explicitly satisfy the constraints.

\section{References}

[1] Arndt M. and Griebel M. (2005). "Derivation of higher order gradient continuum models from atomistic models for crystalline solids", Multiscale Mod. Simul., 4(2), p. 531-562. 
[2] Arndt M. and Luskin M. (2007). "Goal-oriented atomistic-continuum adaptivity for the quasicontinuum approximation", International Journal for Multiscale Computational Engineering, 5, p. 407-415.

[3] Arndt M. and Luskin M. (2008). "Error estimation and atomistic-continuum adaptivity for the quasicontinuum approximation of a Frenkel-Kontorova model", SIAM J. Multiscale Modeling \&S Simulation, 7, p. 147-170.

[4] Badia S., Bochev P., Fish J., Gunzburger M., Lehoucq R., Nuggehally M., and Parks M. (2007). "A force-based blending model for Atomistic-to-Continuum coupling", International Journal for Multiscale Computational Engineering, 5(5), p. 387-406.

[5] Badia S., Parks M., Bochev P., Gunzburger M., and Lehoucq R. (2008). "On atomistic-to-continuum coupling by blending", Multiscale Modeling 83 Simulation, 7(1), p. 381-406.

[6] Bauman P. T., Dhia H. B., Elkhodja, N., Oden J. T., and Prudhomme S. (2008). "On the application of the Arlequin method to the coupling of particle and continuum models", Computational Mechanics. Article in Press.

[7] Bažant, Zdeněk P. and Jirásek M. (2002). "Nonlocal integral formulations of plasticity and damage: Survey of progress", J. of Eng. Mech., 128, p. 1119 1149.

[8] Belytschko T. and Xiao S. P. (2003). "Coupling methods for continuum model with molecular model", International Journal for Multiscale Computational Engineering, 1(1), p. 115-126.

[9] Blanc, Xavier, Bris, Claude Le, and Lions, P.-L. (2007). "Atomistic to continuum limits for computational materials science", Math. Model. Num. Anal., 41(2), p. 391-426.

[10] Born M. and Huang K. (1954). Dynamical Theory of Crystal Lattices, Clarendon Press.

[11] Brezzi F. and Fortin M. (1991). Mixed and hybrid finite element methods, Springer, Verlag.

[12] Chen Y. and Lee J. (2005). "Atomistic formulation of a multiscale field theory for nano/micro solids", Philosophical Magazine, 85(33-35), p. 40954126.

[13] Chen Y., Lee J. D., and Eskandarian A. (2004). "Atomistic viewpoint of the applicability of microcontinuum theories", Int. J. of Solids and Structures, 41, p. 2085-2097. 10.1016/j.ijsolstr.2003.11.030.doi

[14] Curtin W. A. and Miller R. E. (2003). "Atomistic/continuum coupling methods in multi-scale materials modeling", Modeling and Simulation in Materials Science and Engineering, 11(3), R33-R68.

[15] de Sciarra, Francesco Marotti (2008). "Variational formulations and a consistent finite-element procedure for a class of nonlocal elastic continua", International Journal of Solids and Structures, 45, p. 4184-4202.

[16] Dhia H. B. and Rateau G. (2005). "The Arlequin method as a flexible engineering design tool", International Journal for Numerical Methods in Engineering, 62, p. 1442-1462. 
[17] Dobson M. and Luskin M. (2008). "Analysis of a force-based quasicontinuum approximation", Mathematical Modelling and Numerical Analysis, 42, p. $113-139$.

[18] E W. and Ming P. (2007). "Cauchy-Born rule and the stability of crystalline solids: Static problems", Archives for Rational Mechanics and Analysis, $\mathbf{1 8 3}(2)$, p. 241-297.

[19] Eringen, A. Cemal (2002). Nonlocal continuum field theories, Springer-Verlag, NewYork, Inc.

[20] Fish J., Nuggehally M. A., Shephard M. S., Picu C. R., Badia S., Parks M. L., and Gunzburger M. (2007). "Concurrent AtC coupling based on a blend of the continuum stress and the atomistic force", Computer Methods in Applied Mechanics and Engineering, 196, p. 4548-4560.

[21] Gao, Huajian and Klien, Patrick (1998). "Numerical simulation of crack growth in an isotropic solid with randomized internal cohesive bonds", J. Mech. Phys. Solids, 46, p. 187-218.

[22] Huerta A., Belytschko T., Fernández-Médez S., and Rabczuk, Timon (2004). Meshfree methods. In Encyclopedia of Computational Mechanics (ed. Stein E., de Borst R., and Hughes T. J. R.), Volume 1 of Fundamentals, Chapter 10, Wiley.

[23] Kirchner N. and Steinmann P. (2007). "Mechanics of extended continua: modeling and simulation of elastic microstretch materials", Comput. Mech., 40.

[24] Klein P. A. and Zimmerman J. A. (2006). "Coupled atomistic-continuum simulations using arbitrary overlapping domains", Journal of Computational Physics, 213, p. 86-116.

[25] Knap J. and Ortiz M. (2001). "An analysis of the quasicontinuum method", J. Mech. Phys. Solids, 49, p. 1899-1923.

[26] Lin P. (2003). "Theoretical and numerical analysis for the quasi-continuum approximation of a material particle model", Mathematics of Computation, 72, p. 657-675.

[27] Lin P. (2007). "Convergence analysis of a quasi-continuum approximation for a two-dimensional material without defects", SIAM Journal on Numerical Analysis, 45(1), p. 313-332.

[28] Miller R. and Tadmor E. (2002). "The quasicontinuum method: Overview, applications and current directions", J. Comput. Aided Mater. Des., 9, p. 203-239.

[29] Miller R. E. and Tadmor E. B. (2007, November). Hybrid continuum mechanics and atomistic methods for simulating materials deformation and failure. In Multiscale Modeling in Advanced Materials Research (ed. J. J. de Pablo and W. A. Curtin), Volume 32 of MRS Bulletin, p. 920-926.

[30] Murdoch A. I. and Bedeaux D. (1994). "Continuum equations of balance via weighted averages of microscopic quantities", Proc. Roy. Soc. London A, 445, p. $157-179$.

[31] Ortner, Christoph and Süli, Endre (2008). "Analysis of a quasicontinuum method in one dimension", M2AN Math. Model. Numer. Anal., 42(1), p. 57-91. 
[32] Parks M. L., Bochev P. B., and Lehoucq R. B. (2008). "Connecting atomisticto-continuum coupling and domain decomposition", Multiscale Modeling 86 Simulation, 7(1), p. 362-380.

[33] Parks M. L., Lehoucq R. B., Plimpton S. J., and Silling S. A. (2008). Implementing peridynamics within a molecular dynamics code. Technical Report SAND2007-7957J, Sandia National Laboratories, Albuquerque, New Mexico 87185 and Livermore, California 94550, 179(11), p. 777-783.

[34] Prudhomme S., Dhia H. B., Bauman P. T., Elkhodja N., and Oden, J. Tinsley (2008). "Computational analysis of modeling error for the coupling of particle and continuum models by the Arlequin method", Computer Methods in Applied Mechanics and Engineering, 197(41-42), p. 3399-3409.

[35] Rogula D. (1982). Introduction to nonlocal theory of material media. In Nonlocal theory of material media (ed. D. Rogula), Number 268 in CISM Courses and Lectures, p. 125-222. Springer Verlag, Wien.

[36] Silling S. A. (2000). "Reformulation of elasticity theory for discontinuities and long-range forces", J. Mech. Phys. Solids, 48, p. 175-209.

[37] Silling S. A., Epton M., Weckner O., Xu J., and Askari E.(2007). "Peridynamic states and constitutive modeling", J. Elasticity, 88, p. 151-184.

[38] Silling, S. A. and Lehoucq, R. B. (2008). "Convergence of peridynamics to classical elasticity theory", J. Elasticity, 93(1), p. 13-37.

[39] Tadmor E. B., Ortiz M., and Phillips R. (1996). "Quasicontinuum analysis of defects in solids". Philosophical Magazine A, 73, p. 1529-1563.

[40] Toselli, Andrea and Widlund, Olof B. (2005). Domain Decomposition Methods-Algorithms and Theory, Volume 34 of Springer Series in Computational Mathematics. Springer.

[41] Wagner G. J. and Liu W. K. (2003). "Coupling of atomic and continuum simulations using a bridging scale decomposition", Journal of Computational Physics, 190, p.249-274.

[42] Zhou Min (2005). "Thermomechanical continuum representation of atomistic deformation at arbitrary size scales", Proceedings of The Royal Society A, 461, p. 3437-3472. 10.1098/rspa.2005.1468.

[43] Zhou, Min and McDowell, David L. (2002). "Equivalent continuum for dynamically deforming atomistic particle systems", Philosophical Magazine A, 82, p. 2547-2574. 
-

JFISH: "CHAP06" - 2009/6/4 - 17:33 — PAGE $190-\# 26$ 